\title{
Post-treatment haemolysis in African children with hyperparasitaemic falciparum malaria; a randomized comparison of artesunate and quinine
}

C. Fanello ${ }^{1,2^{*}}$, M. Onyamboko ${ }^{3}$, S. J. Lee ${ }^{1,2}$, C. Woodrow ${ }^{1,2}$, S. Setaphan ${ }^{1}$, K. Chotivanich ${ }^{1,4}$, P. Buffet B, $^{5,7}$,

S. Jauréguiberry ${ }^{7}$, K. Rockett $^{8}$, K. Stepniewska ${ }^{2,9}$, N. P. J. Day ${ }^{1,2}$, N. J. White ${ }^{1,2}$ and A. M. Dondorp ${ }^{1,2}$

\begin{abstract}
Background: Parenteral artesunate is the treatment of choice for severe malaria. Recently, haemolytic anaemia occurring 1 to 3 weeks after artesunate treatment of falciparum malaria has been reported in returning travellers in temperate countries.

Methods: To assess these potential safety concerns in African children, in whom most deaths from malaria occur, an open-labelled, randomized controlled trial was conducted in Kinshasa, Democratic Republic of Congo. 217 children aged between 6 months and 14 years with acute uncomplicated falciparum malaria and parasite densities over 100,000/ $\mu \mathrm{L}$ were randomly allocated to intravenous artesunate or quinine, hospitalized for 3 days and then followed for 42 days.

Results: The immediate reduction in haemoglobin was less with artesunate than with quinine: median (IQR) fall at $72 \mathrm{~h} 1.4 \mathrm{~g} / \mathrm{dL}(0.90-1.95)$ vs. $1.7 \mathrm{~g} / \mathrm{dL}(1.10-2.40)(p=0.009)$. This was explained by greater pitting then recirculation of once infected erythrocytes. Only $5 \%$ of patients (in both groups) had a $\geq 10 \%$ reduction in haemoglobin after day $7(p=0.1)$. One artesunate treated patient with suspected concomitant sepsis had a protracted clinical course and required a blood transfusion on day 14 .
\end{abstract}

Conclusions: Clinically significant delayed haemolysis following parenteral artesunate is uncommon in African children hospitalised with acute falciparum malaria and high parasitaemias.

Trial registration: ClinicalTrials.gov; Identifier: NCT02092766 (18/03/2014)

\section{Background}

Parenteral treatment with artesunate has been shown to reduce mortality from severe malaria by $35 \%$ in adults and $23 \%$ in children compared to quinine $[1,2]$ and is the recommended first-line treatment for severe malaria [3]. Artesunate is well tolerated, safe and has fewer adverse effects than quinine. Until recently the only serious adverse event observed has been a Type-1 hypersensitivity reaction in approximately one per 3000

\footnotetext{
* Correspondence: caterina@tropmedres.ac

${ }^{1}$ Mahidol-Oxford Tropical Medicine Research Unit, Faculty of Tropical

Medicine, Mahidol University, Bangkok, Thailand

${ }^{2}$ Centre for Tropical Medicine and Global Health, Nuffield Department of

Medicine, University of Oxford, Oxford, UK

Full list of author information is available at the end of the article
}

treatments [4-6]. However, there are now several reports, mainly from temperate countries, of late haemolytic anaemia occurring 1 to 3 weeks after parenteral artesunate treatment of hyperparasitaemic falciparum malaria in returned travellers. This has raised general concerns about the safety of these life-saving compounds. Most cases followed intravenous artesunate, although some were reported after intramuscular artemether, intrarectal artesunate and oral artemisinin derivatives $[7,8]$. There were no deaths, but blood transfusion was often required. A recent review of published data, from non-immune and semi-immune patients, estimated the incidence of late haemolysis after intravenous artesunate to be $13 \%$ (95\% CI 9-18\%) and the 
requirement for a blood transfusion at 9\% (95\% CI 614\%) [7], although most data are from case reports and case definitions have varied substantially. But returned travellers constitute only a very small fraction of the global burden of severe malaria. Are these observations in travellers of clinical relevance to African children, who carry more than $90 \%$ of the global malaria disease burden, and for whom life-threatening anaemia is a common clinical presentation of falciparum malaria? Severe malarial anaemia in childhood is associated with significant mortality both in hospital and after discharge [9]. As haemolytic anaemia is the pathological hallmark of all malaria infections, and severe anaemia is a common feature of severe malaria, dissecting disease from drug-associated mechanisms is difficult. To investigate the pathogenesis of anaemia in African children and whether post-artesunate haemolytic anaemia is a significant problem, a randomised comparison was conducted of the immediate and delayed haematological responses to parenteral artesunate and quinine in children hospitalised with high falciparum parasitaemias.

\section{Methods}

The trial was conducted at the Kinshasa Mahidol Oxford Research Unit, Kinshasa, the Democratic Republic of Congo. Malaria in this area is endemic and perennial, with minor seasonal variations.

Children with acute falciparum malaria were eligible for enrolment if they were aged between 6 months and 14 years old, had body weight $>5 \mathrm{~kg}$, haemoglobin $\geq 5.0 \mathrm{~g} / \mathrm{dL}$ and parasitaemia $\geq 100,000$ falciparum parasites $/ \mu \mathrm{L}$. Patients with any sign of severe malaria [3] (although hyperparasitaemia alone was not an exclusion criterion [10]), a history of hypersensitivity or contraindication to the use of artesunate or quinine, a clear history of adequate antimalarial treatment within $24 \mathrm{~h}$, or the presence of a significant other illness, were excluded. The study was explained to caregivers in French or Lingala and they were requested to sign a consent form to allow their child's participation in the study.

The study was an open-labelled randomised comparison of the hematological effects of parenteral antimalarial treatment with either artesunate or quinine. Computer randomisation, in blocks of 20 , was prepared by a study statistician. Treatment allocation was concealed in sequential opaque envelopes prepared by an independent person. The intervention was assigned by the study nurse, after the doctor confirmed eligibility and the caregivers had signed the informed consent. After a full clinical examination, patients were randomised to receive intravenous (IV) artesunate (AS) $2.4 \mathrm{mg} / \mathrm{kg}$ body weight bolus (Guilin Pharmaceutical, Guangxi, China) immediately and at $12,24,48$ and $72 \mathrm{~h}$ or quinine dihydrochloride (QN) $20 \mathrm{mg}$ salt/kg body weight (Sterop, Brussels, Belgium) infused over $4 \mathrm{~h}$, followed by $10 \mathrm{mg} / \mathrm{kg}$ body weight every $8 \mathrm{~h}$ infused over $2 \mathrm{~h}$ until $72 \mathrm{~h}$.

Parenteral treatment was followed by a full course of oral artemether-lumefantrine (Coartem ${ }^{\circ}$, Novartis); the first dose was directly observed and the remaining doses were given with instructions to the caregiver for administration at home. Patients were followed-up weekly for 6 weeks. Any recurrent episodes of malaria were treated with fixed-dose amodiaquine-artesunate tablets (Winthrop $^{\circ}$, Sanofi). Blood transfusion was given when haemoglobin was $\leq 5 \mathrm{~g} / \mathrm{dL}$ regardless of clinical symptoms, or if the haemoglobin was $\leq 7 \mathrm{~g} / \mathrm{dL}$ and there were one or more clinical features of severity present [3].

Malaria blood films were prepared at admission, 0 (baseline), 6, $12 \mathrm{~h}$, then every $12 \mathrm{~h}$ until 2 consecutive microscopy negative blood films and at each follow-up visit. Parasites were counted using standard methods. Hemoglobin $(\mathrm{Hb})$ and haematocrit $(\mathrm{Hct})$ were assessed at the same time-points as the blood films using HemoCue Hb201+ (Angelholm, Sweden) and Hawksley Haematospin 1400 (Hawksley \& Sons, Ltd. UK). Reticulocytes were counted as a percentage of total RBCs at $0,72 \mathrm{~h}$ and weekly.

Ring-infected Erythrocyte Surface Antigen (RESA)-positive, falciparum-negative RBCs (once infected or oi-RBCs) were counted per $1000 \mathrm{RBCs}$ at $0,24,48,72 \mathrm{~h}$ and then weekly by immunofluorescence microscopy [11]. Total and differential white blood cell (WBC) counts were measured at $0,72 \mathrm{~h}$ and weekly by QBC Star ${ }^{\mathrm{mw}}$.

Hemoglobin HbS [rs334] and $\mathrm{A}^{-}$glucose-6-phosphate dehydrogenase deficiency defined by G6PD 376 (rs1050829) and G6PD 202 (rs1050828) were characterised from genomic DNA at the Wellcome Trust Centre for Human Genetics using Agena MassArray IPLEX platform and multiplexed PCR assay [12].

Quantification of plasma PfHRP2 by ELISA (Cell-labs, Australia) and plasma lactate dehydrogenase (LDH; Olympus AU400 Automated chemistry analyser) was performed in Bangkok, Thailand. Laboratory technicians were blinded to study treatments.

The prespecified primary endpoint was the proportion of patients who developed late anaemia, defined as a reduction of $\geq 10 \%$ in haemoglobin compared with any previous measurement between days 7 and 42 after starting parenteral antimalarial treatment. Secondary endpoints included: the proportions of patients requiring blood transfusion, the fractional decreases in haemoglobin in the first week of treatment, plasma LDH as a measure of haemolysis, reticulocyte and pitted erythrocyte counts, parasite clearance times, and frequency of serious adverse events.

The proportion of intravenous artesunate treated patients with delayed anaemia was assumed to be $20 \%$ [13]. To provide $90 \%$ power and $95 \%$ confidence to 
detect a difference of $20 \%$ vs. $4 \%$, with $10 \%$ loss to follow-up, 108 children per arm were recruited.

Comparisons between groups used the Student's t-test or Mann-Whitney $U$ test, depending on distributions. Kendall's Tau ( $\tau$ ) or Pearson's correlation coefficient $\left(\right.$ rho $_{\mathrm{p}}$ ) assessed associations between continuous variables. For more than two groups, the Kruskal-Wallis test was used. AUCs were estimated using the trapezoid rule. Hemoglobin, oi-RBC and parasite count values were censored on the day of malaria recrudescence or blood transfusion.

Parasite clearance was assessed as the time for the parasite count to fall by $50 \%\left(\mathrm{PC}_{50}\right)$, the parasite clearance half-life $\left(\mathrm{PC}_{1 / 2}\right)$ [14] and the interval until patients became parasite negative by microscopy (PCT).

Clearance of $o i$-RBC was estimated by modelling the decline in log-transformed oi-RBC over time in a random-effects model, with variable intercept and slope. Mono- and biexponential decay curves were considered. The final model was selected based on the Akaike Information Criterion (AIC). Levels of oi-RBC below the detection limit were excluded from the analysis.

To quantify associations with significant predictors of haemoglobin concentration, linear regression was used with random effects fitted for both the intercepts and slopes of $\mathrm{Hb},[\log ]$ oi-RBC, LDH and [log] reticulocytes. Fixed covariates included sex, age, homozygous/hemizygous G6PD deficiency, sickle cell disease trait, [log] parasitaemia on admission, splenomegaly on admission, malnutrition (a composite of underweight, wasted and stunted) and study drug treatment. Two models were considered: the first to evaluate associations with haemoglobin changes from day 0 to 7 (which included $\mathrm{Hb}$ at hour 0 as a covariate), the second to evaluate associations from day 7 to 28 .

Safety reporting was performed according to the $\mathrm{ICH}$ Harmonized Tripartite Guideline for GCP (1996). The study was approved by the Oxford Tropical Medicine Ethics Committee and the University of Kinshasa, School of Public Health institutional review board. Data were entered using MACRO InferMed and analysed using STATA v14.0. (ClinicalTrials.gov; Identifier: NCT02092766).

\section{Results}

Between June 2014 and March 2015, 217 children were enrolled (Fig. 1). One study patient, whose parents withdrew their consent the first day of hospitalization, was

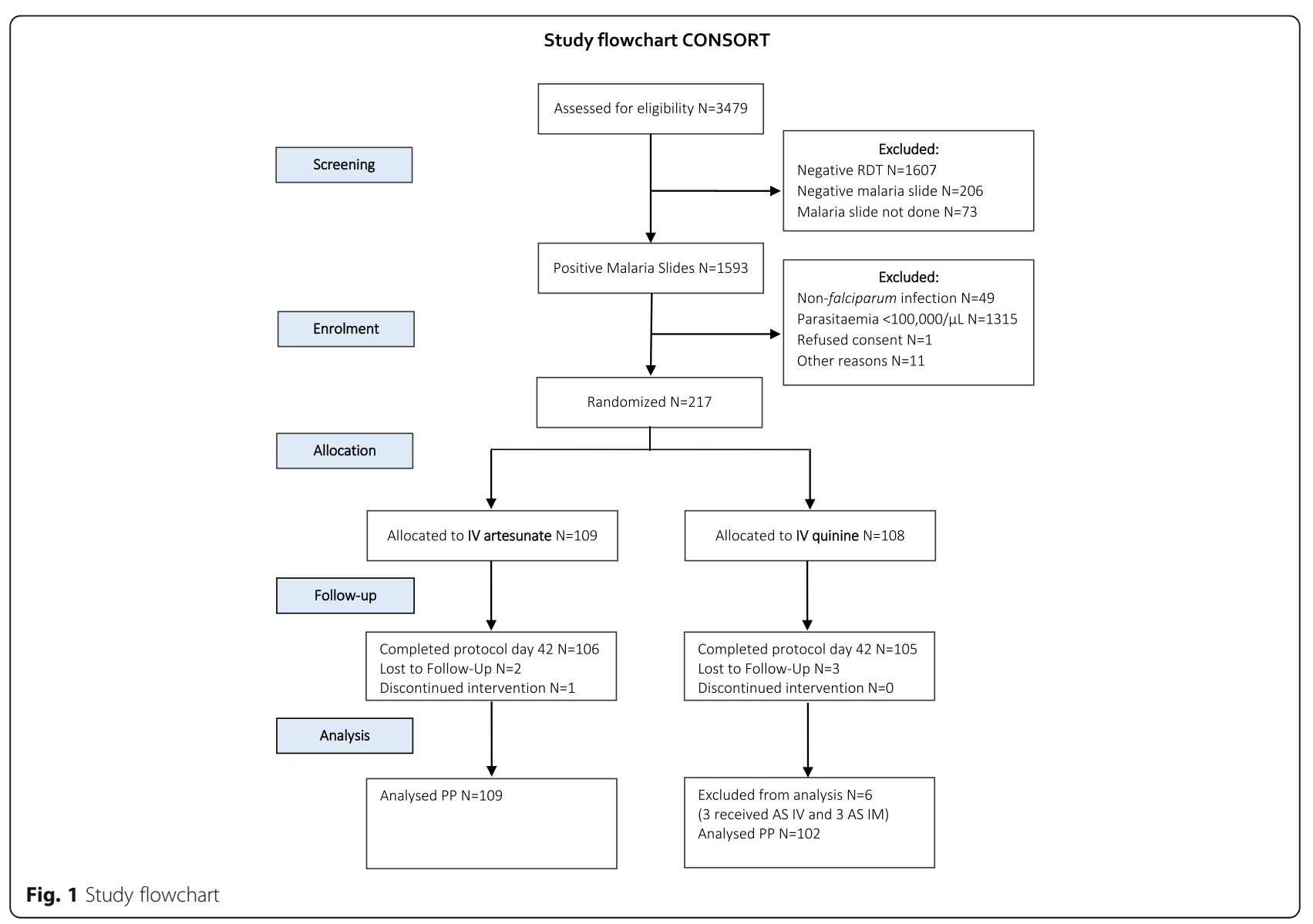


replaced (AS). Four patients developed severe malaria in the first 24 h (AS 1; QN 3); for those allocated to QN, the treatment was changed to IV AS. In three other cases (QN), difficulties in obtaining venous access prompted a change to intramuscular (IM) artesunate. Thus, hematological outcomes were analysed in 211 children (AS 109; QN 102). The two groups had similar demographic, clinical and laboratory characteristics on admission (Table 1). Five patients were lost to follow-up after day 7 (AS 2; QN 3), all remaining patients were followed until day 42. Most patients (75.6\%) had an adequate clinical and parasitological response at day 42; forty-one (18.9\%) had a recurrent episode of malaria with a similar rate in the two arms (log-rank test $p=0.80$ ) (Additional file 1: Table S1).

\section{Dynamics of infected and once-infected erythrocytes}

Admission geometric mean parasitaemias were similar in the two treatment groups (Table $1 ; p=0.89$ ) and correlated inversely with age $\left(\mathrm{rho}_{\mathrm{p}}=-0.186, p=0.007\right)$. Parasites cleared significantly faster in patients treated with AS compared to QN: $\mathrm{PC}_{50}$ median (range) $5.1 \mathrm{~h}$ (0.08-15) vs. QN 15 h (0.04-28.6), $p=0.0001 ; \mathrm{PC}_{1 / 2}$ median (range) AS $1.8 \mathrm{~h}(1.0-3.2)$ vs. QN $2.8 \mathrm{~h}$ (1.09.7), $p=0.0001$. The median (IQR) PCT was 2 days in the AS arm (1-2) vs. 3 days in the QN arm (2-3), $p<0.001$.

The oi-RBC counts peaked at $24 \mathrm{~h}$ in the AS group and at $48 \mathrm{~h}$ in the QN group (IQR 1-2 days for both arms; $p=0.09$; Fig. $2 \mathrm{~b}$ ). The median post-treatment oiRBC peak was over three times higher with AS compared to QN: $100,417 / \mu \mathrm{L}$ vs. $31,714 / \mu \mathrm{L}(p<0.001$, Additional file 1: Table S2). The ratio between maximum number of $o i-\mathrm{RBC}$ and baseline parasitaemia was $59 \%$ with AS vs. $14 \%$ with QN $(p<0.001)$.

A first-order biexponential decay best described the decline in $o i-\mathrm{RBC}$ values: the initial decline was similar between treatments with a half-life of 2.0 (95\% CI $1.7-$ 2.3) days. The terminal elimination phase began around 2 days after peak values with a half-life which was shorter following AS than QN: 10.0 (95\% CI 9.0-11.1) days vs.14.8 (95\% CI 12.6-18.0) days $(p<0.001)$.

Table 1 Demographic, clinical and parasitological data at admission by treatment arm (as per allocation)

\begin{tabular}{|c|c|c|}
\hline & AS & QN \\
\hline Number of admitted patients & 109 & 108 \\
\hline Female: Male & $59: 50$ & $52: 56$ \\
\hline Mean (range) age (months) & $70.3(9-167)$ & $66.8(6-153)$ \\
\hline Mean (SD) weight (kg) & $18.6(7.8)$ & $18.0(6.8)$ \\
\hline Mean (SD) height (cm) & $109.5(21.8)$ & $107.7(20.6)$ \\
\hline Malnourished (\%) & 31.2 & 23.5 \\
\hline$A^{-}$G6PD allele frequency (\%) & 18.3 & 15.7 \\
\hline Sickle cell trait allele frequency (\%) & 6.9 & 6.9 \\
\hline Palpable splenomegaly (\%) & 49.5 & 40.7 \\
\hline Hepatomegaly (\%) & 28.4 & 22.2 \\
\hline Median (range) Axillary temperature $\left({ }^{\circ} \mathrm{C}\right)$ & $37.1^{\circ} \mathrm{C}(36.0-40.4)$ & $37.0^{\circ} \mathrm{C}(36.0-39.9)$ \\
\hline Mean (SD) Systolic blood pressure (mmHg) & $89 \pm 8$ & $89 \pm 9$ \\
\hline Mean (SD) Diastolic blood pressure (mmHg) & $54 \pm 8$ & $53 \pm 8$ \\
\hline Mean (SD) Heart Rate (beats/ min) & $125 \pm 21$ & $123 \pm 21$ \\
\hline Mean (SD) Respiratory Rate (breaths/min) & $36 \pm 8$ & $34 \pm 6$ \\
\hline Mean (SD) WBC count, $10^{3} / \mathrm{ML}$ & $8.0(3.5)$ & $7.3(3.1)$ \\
\hline Mean (SD) haemoglobin, g/dL & $10.4(1.7)$ & $10.3(1.8)$ \\
\hline Mean (SD) haematocrit (\%) & $31.3(4.9)$ & $30.7(5.3)$ \\
\hline Median P. falciparum parasitaemia/ $\mu \mathrm{L}$ (range) & $\begin{array}{l}179,608 \\
(100,480-1,386,624)\end{array}$ & $\begin{array}{l}177,473 \\
(101,108-840,264)\end{array}$ \\
\hline Geometric mean P. falciparum density $(/ \mu \mathrm{L})(95 \% \mathrm{Cl})$ & $\begin{array}{l}201,450 \\
(181,764-223,268)\end{array}$ & $\begin{array}{l}199,070 \\
(181,496-218,346)\end{array}$ \\
\hline No. patients with >250,000/ML (\%) & $35.8 \%$ & $41.7 \%$ \\
\hline Geometric mean plasma PfHRP2 ng/mL (95\% Cl) & $\begin{array}{l}558.8(N=51) \\
(369.7-844.6)\end{array}$ & $\begin{array}{l}564.7(N=47) \\
(344.7-925.1)\end{array}$ \\
\hline Median oi-RBC count/ML (range) & $\begin{array}{l}10,927 \\
(0-104,499)\end{array}$ & $\begin{array}{l}11,680 \\
(0-145,947)\end{array}$ \\
\hline
\end{tabular}



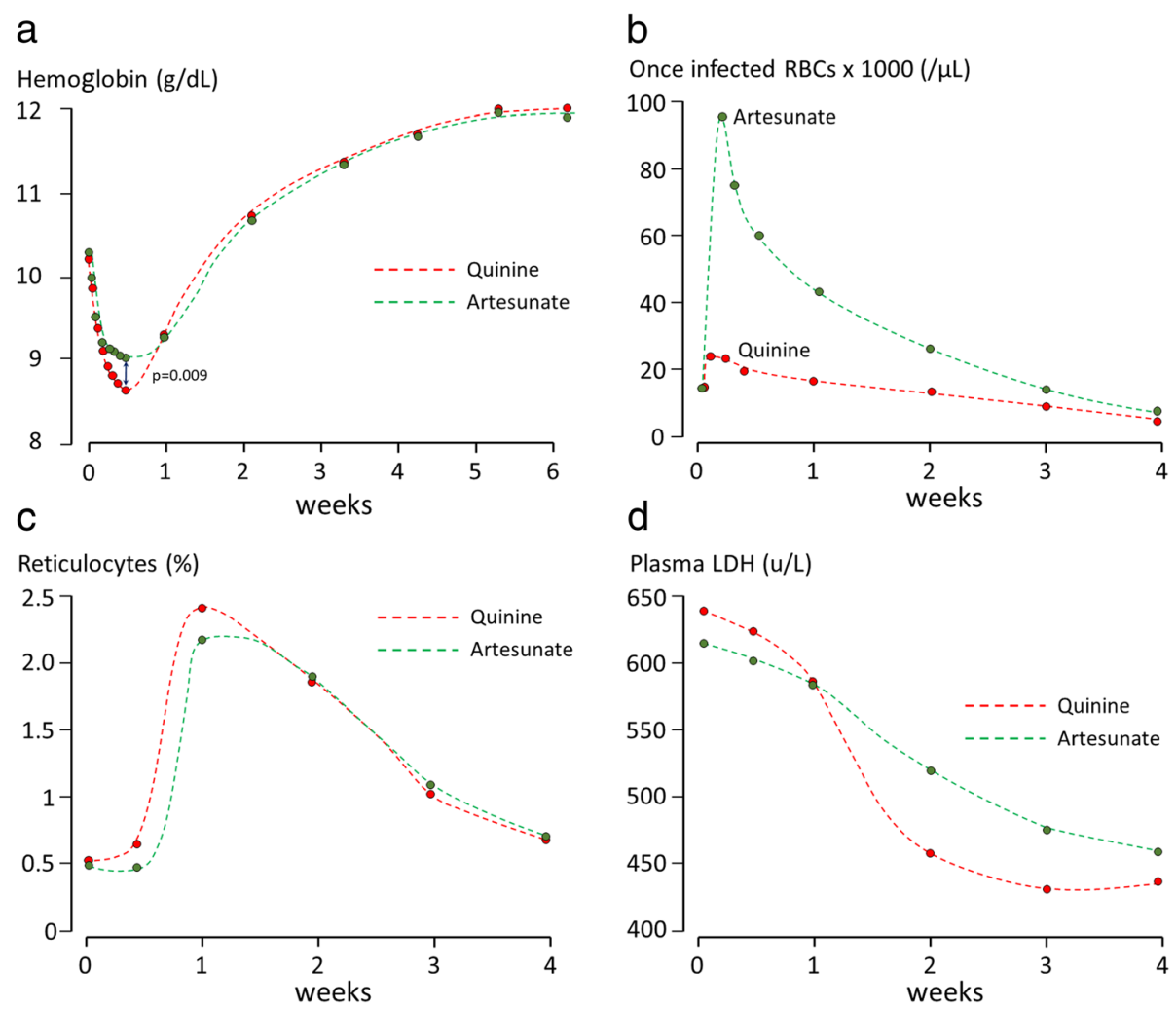

Fig. 2 Mean haemoglobin a, median oi-RBCs b, reticulocyte counts $\mathbf{c}$ and LDH $\mathbf{d}$, by treatment, over time

\section{Anaemia}

On admission $74 \%$ of children were anaemic according to WHO cut-offs by age-group [15]: $23 \%$ mild, $47 \%$ moderate and $4 \%$ severe. The mean haemoglobin concentration was similar in the two arms (Additional file 1: Table S3 \& Fig. 2a, p=0.600) and correlated positively with age $\left(\right.$ rho $\left._{p}=0.36, p<0.001\right)$. Malnourished children had a significantly lower mean (SD) Hb $9.7 \mathrm{~g} / \mathrm{dL}$ (1.7) vs. $10.4 \mathrm{~g} / \mathrm{dL}$ (1.6); $p=0.006$. Children homozygous/hemizygous for G6PD deficiency $(N=22)$ also had a lower mean (SD) values, $9.7 \mathrm{~g} / \mathrm{dL}(1.4)$ vs. $10.3 \mathrm{~g} / \mathrm{dL}(1.7)$, although the difference was not significant $(p=0.12)$.

Four patients received blood transfusions, two in the first $12 \mathrm{~h}(\mathrm{QN})$, one at day 5 (AS) and one at day 14 (AS). The single case given a late blood transfusion was a 6year-old boy admitted with a parasitaemia of $145,068 / \mu \mathrm{L}$ which increased abruptly to $887,992 / \mu \mathrm{L}$ in the $25 \mathrm{~min}$ interval to the start of treatment (suggesting highly synchronous schizogony). The following day the patient deteriorated, was unable to sit unaided and became deeply jaundiced; antibiotics were started for suspected bacterial sepsis. During two-weeks' hospitalisation, his condition slowly improved, but fever persisted and the antibiotics were changed to ceftriaxone. His haemoglobin declined steadily from $11.0 \mathrm{~g} / \mathrm{dL}$ on admission to $4.7 \mathrm{~g} / \mathrm{dL}$ on day 14 , with a reticulocyte count of $9.6 \%$ and plasma LDH of
2126 U/L. The patient was given a blood transfusion and discharged. He was clinically well when reviewed a week later.

The initial reduction in haemoglobin was greater in the QN group: median (IQR) $1.5 \mathrm{~g} / \mathrm{dL}(0.90-2.20)$ vs. $1.3 \mathrm{~g} / \mathrm{dL}(0.80-1.90)$ by $48 \mathrm{~h}(p=0.036)$, and $1.7 \mathrm{~g} / \mathrm{dL}$ $(1.10-2.40)$ vs. $1.4 \mathrm{~g} / \mathrm{dL}(0.90-1.95)$ by $72 \mathrm{~h}(p=0.009)$. However, by day 7 the haemoglobin values were similar in the two treatment arms (Fig. 2a).

The $\mathrm{Hb}$ nadir was observed before day 7 in $88 \%$ of patients $(N=185$, lowest recorded $\mathrm{Hb} 4.5 \mathrm{~g} / \mathrm{dL})$ and at day 7 in $10 \%(N=21$, lowest recorded $\mathrm{Hb} 7.3 \mathrm{~g} / \mathrm{dL})$. The median (IQR) interval to $\mathrm{Hb}$ nadir was $48 \mathrm{~h}(36-72)$ with $\mathrm{AS}$ and $60 \mathrm{~h}(48-72)$ with QN, $p=0.192$. The mean (SD) $\mathrm{Hb}$ nadir values before day 7 were similar, AS $=8.3(1.4) \mathrm{g} / \mathrm{dL}$ vs. $\mathrm{QN}=8.1$ (1.6) $\mathrm{g} / \mathrm{dL}, p=0.395$, as were the nadirs observed at day 7 , AS $=9.2(1.1) \mathrm{g} / \mathrm{dL}$ vs. $\mathrm{QN}=9.4(1.2) \mathrm{g} / \mathrm{dL}, p=0.736$.

Reticulocyte counts (haematocrit corrected) increased until day 7 and then decreased gradually in both arms (Fig. 2c). The counts were significantly higher on day 3 in children who received QN, $p<0.001$. There were no significant differences after day 3 .

The primary endpoint, defined a-priori as a reduction of $\geq 10 \%$ in the $\mathrm{Hb}$ level at any time between days 7 and 42, was observed in 19/207 patients: 10 children treated 
with AS, whose median $\mathrm{Hb}$ fractional reduction was $13.7 \%$ (IQR 12.7-20.7; lowest $\mathrm{Hb} 4.7 \mathrm{~g} / \mathrm{dL}$ ) and 9 treated with $\mathrm{QN}$, whose median $\mathrm{Hb}$ fractional reduction was $12.8 \%$ (IQR 11.1-13.4; lowest $\mathrm{Hb} 9.4 \mathrm{~g} / \mathrm{dL}, p=0.103$; Additional file 2: Figure S1 and Additional file 3: Figure S2).

In the cohort of patients who completed the 42-days follow-up, the $\mathrm{Hb}$ concentration increased steadily and rose above the admission level in $92.7 \%$ of cases (AS 94/ 105 vs. QN 96/100; $p=0.08)$. In 15 patients $(7.3 \%)$ the $\mathrm{Hb}$ remained below the admission value throughout the follow-up (AS 11; QN 4) and in 4 patients (2\%) the nadir occurred after day 7 (AS 3; QN 1; lowest Hb 4.7 and $10.4 \mathrm{~g} / \mathrm{dL}$, respectively).

\section{Measures of haemolysis}

Between day 0 and 7 the median plasma LDH concentrations (U*days/L) decreased by similar amounts in the two groups (Fig. 2d): AUCs AS 4224 vs. QN 4380.5 $(p=0.73)$. The median (range) maximum LDH concentration was 688 (328-2215) U/L with AS and 703 (3751664) U/L with QN.

From day 7 onward the median LDH concentration decreased more in patients who received QN $(p<0.05$, Fig. 2d). The median (IQR) fractional reduction from day 7 to 14 was $19.4 \%$ in the $\mathrm{QN}$ arm $(6.5-27.7)$ and $11.8 \%$ in the AS arm (0.2-20.9); $p=0.005$; and between day 14 and $21,24.1 \%$ in the QN arm (12.5-35.3) and $17.9 \%$ in the AS arm (6.4-32.4); $p=0.12$. The median LDH AUC was slightly smaller with QN compared to AS (9863 vs. 10,$637 ; p=0.04)$ and was positively correlated with the maximum oi-RBC count with AS $(p=0.045)$, but not $\mathrm{QN}(p=0.263)$.

Some patients had transient fluctuations in the plasma LDH level during the follow-up period. A significant increase (above twice the paediatric reference range ULN [16]) was observed in $21 \%(23 / 109)$ of AS and $12.7 \%$ of QN treated patients $(13 / 102, p=0.107)$.

Children who received AS and later had a significantly elevated plasma LDH concentration were younger $(p=0.045)$, had higher admission parasitaemias $(p=0.0042)$, higher maximum oi-RBC counts $(p=0.0150)$, higher reticulocyte counts $(p=0.0102)$ and lower $\mathrm{Hb}$ at days 14 and 21 ( $p=0.0004$ and $p=0.0139$, respectively). In the QN arm there were no differences in these parameters between patients with and without significant LDH elevations, although oi-RBC counts were higher in those with normal LDH $(p=0.0132)$.

\section{Factors affecting anaemia}

Factors affecting the change in haemoglobin were assessed by mixed effects modelling. Compared with the remaining patients, children homozygous/hemizygous for G6PD deficiency had an average $0.687 \mathrm{~g} / \mathrm{dL}$ (95\% CI $0.145-1.229)$ lower $\mathrm{Hb}$ values from day 0 to 7 , those with palpable splenomegaly had $0.431 \mathrm{~g} / \mathrm{dL}$ (95\% CI 0.113-0.749) lower $\mathrm{Hb}$ and malnourished children had $0.379 \mathrm{~g} / \mathrm{dL}$ lower $\mathrm{Hb}$ levels (95\% CI 0.024-0.734). Older children had higher $\mathrm{Hb}$ levels, $0.111 \mathrm{~g} / \mathrm{dL}$ (95\% CI 0.055-0.161) per year of age. The effect of treatment was borderline: $\mathrm{Hb}$ levels in children treated with AS were, on average, $0.312 \mathrm{~g} / \mathrm{dL}$ (95\% CI $0.002-0.627$ ) higher from day 0 to 7 , but thereafter the effect of treatment was no longer significant $(p=0.087)$ and only LDH, $[\log ]$ oi-RBC and $[\log ]$ reticulocytes were associated significantly with haemoglobin changes (Additional file 1: Tables S4-S5).

\section{Discussion}

Anaemia is a consistent feature of falciparum malaria and results from the obligatory lysis of infected erythrocytes at schizogony, increased splenic clearance of both infected and uninfected erythrocytes and dyserytropoiesis. The relative contributions of these factors vary with age, immunity and disease severity. In tropical areas malaria anaemia may be compounded by haematinic deficiencies, hypersplenism, and genetic red cell disorders. As severe malarial anaemia can be fatal, it is important to identify preventable contributors. In this study of African children with acute falciparum malaria and high parasite densities living in a high transmission area post-artesunate haemolytic anaemia was substantially less common than reported in non-immune returned travellers.

Post-artesunate haemolytic anaemia has been attributed to the increased pitting of drug-damaged malaria parasites from infected erythrocytes [17]. Pitting is the physiological process whereby the spleen removes intraerythrocytic particulate matter, in this case the pyknotic ring-stage intra-erythrocytic parasites, and then returns the resealed red blood cells into the systemic circulation [11]. The life-saving benefit of artesunate over quinine derives from its rapid parasiticidal activity against the circulating ring-stage parasites. This results in a higher rate of parasite clearance and a correspondingly greater production of oi-RBC (Fig. 2b). These once-infected red blood cells (oi-RBC) have a much shorter survival time (7-14 days) compared with normal erythrocytes in healthy subjects (120 days) or in patients following severe malaria (44 days) $[18,19]$. The shorter survival of $o i-\mathrm{RBC}$ following artesunate probably reflects drug killing and then pitting of developed ring forms whereas background (and quinine associated) pitting may only occur for very young ring stages shortly after merozoite invasion with correspondingly less damage to the erythrocytes. The rapid and synchronous elimination of these $o i$-RBC from the circulation 1 to 2 weeks after the start of antimalarial treatment might result in haemolytic anaemia [17]. 
In this study, a similar proportion of artesunate and quinine treated patients had $\mathrm{a} \geq 10 \%$ reduction in haemoglobin, but the degree of anaemia was moderate, although there are small but significant differences in the time course. Patients receiving artesunate had slightly lower initial reductions in haemoglobin and delayed reticulocyte responses. Previous studies also indicated a clinically insignificant temporary suppression of reticulocytosis by artemisinins in the treatment of malaria [20, 21]. More than three times as many ring stage parasites were pitted after treatment with artesunate compared to quinine, with over half returning to the circulation, largely explaining the initially smaller reduction in haemoglobin in artesunate recipients. The ratio of $o i-\mathrm{RBC}$ to the initial parasitaemia (59\%) was comparable to that observed in Malian children (60\%) following artesunate, but lower than in returned travellers $(78 \%)$ [17]. Moreover, in artesunate-treated travelers around $60 \%$ of originally parasitized cells were still present in the circulation 7 days after treatment, while in this study only approximately one quarter of the oiRBCs were left at this stage, thereby reducing the number of erythrocytes contributing to late haemolysis. These findings suggest that other, presumably immune mediated, mechanisms leading to infected cell removal contribute to splenic clearance of parasitaemia in African children. The erythropoietic response to malarial anaemia was rapid (peak 7 days) [22], and took place earlier than in patients with less background immunity (14-21 days $[17,19,23])$. Hence in these semi-immune African children the lower contribution of pitting to parasite clearance after artesunate and the earlier reticulocyte response mitigated the loss of haemoglobin associated with the late lysis of oi-RBC, whilst more rapid clearance of pitted cells reduced haemolysis occurring after 7 days. Only one artesunate treated patient had haemolytic anaemia severe enough to warrant blood transfusion. This patient had a complicated disease course with suspected concomitant bacterial sepsis treated. Whether the haemolysis in this case was explained only by malaria and artesunate, or whether the presumed sepsis contributed cannot be determined.

Severe delayed haemolytic anaemia was observed in less than $1 \%$ of Congolese children with acute falciparum malaria and high parasitaemias, which is similar to the frequency reported in West African children with severe malaria [22]. A limitation of this study is that the patients were not clinically severe whereas postartesunate delayed haemolysis has been reported particularly after severe malaria. Children with severe malaria could not be randomised because artesunate is clearly the better treatment. Nevertheless, the patients studied were at the severe end of the clinical spectrum of hospitalised uncomplicated malaria. Plasma PfHRP2 concentrations on admission, an indicator of parasite burden, were between the values reported for African children with uncomplicated and severe malaria (approximately $150 \mathrm{ng} / \mathrm{ml}$ and $1500 \mathrm{ng} / \mathrm{ml}$ respectively [24]).

\section{Conclusions}

In conclusion, severe delayed haemolytic anaemia was rare in this population of African children with high parasitaemias. This reassuring evidence contrasts with recent reports form returned travellers and it strongly supports the wider deployment of life-saving parenteral artesunate for the treatment of severe falciparum in African children.

\section{Additional files}

Additional file 1: Table S1. Efficacy outcome by treatment arm PCR uncorrected, Per Protocol. Table S2. Median and range oi-RBCs/ $\mu \mathrm{L}$ of blood by day and treatment arm. Table S3. Mean, SD and range of haemoglobin 0-42 days by treatment arm. Table S4. Linear regression using $\mathrm{Hb}(\mathrm{g} / \mathrm{dL})$ as outcome and patient as random effect for days 0-7. Table S5. Linear regression using $\mathrm{Hb}(\mathrm{g} / \mathrm{dL})$ as outcome and patient as random effect for days 7-28. (DOCX $24 \mathrm{~kb}$ )

Additional file 2: Figure S1. Individual haemoglobin profiles of patients in the QN group with $\geq 10 \%$ reduction after day 7, $n=9$ (TIFF $24 \mathrm{~kb}$ )

Additional file 3: Figure S2. Individual haemoglobin profiles of patients in the AS group with $\geq 10 \%$ reduction after day $7, n=10$ (TIFF $64 \mathrm{~kb})$

\section{Abbreviations}

RESA: Ring-infected erythrocyte surface antigen; 95\% Cl: 95\% Confidence interval; AIC: Akaike information criterion; AS: Artesunate; AUC: Area under the curve; DRC: Democratic Republic of Congo; G6PD: Glucose-6-phosphate dehydrogenase; Hb: Hemoglobin; Hct: Haematocrit; IM: Intramuscular; IQR: Interquartile range; IV: Intravenous; LDH: Plasma lactate dehydrogenase; once infected or oi-RBCs: RESA-positive, falciparum-negative RBCs; $\mathrm{PC}_{1 /}$

2: Parasite clearance half-life; $P C_{50}$ : Time for parasite count to fall by $50 \%$; PCT: Parasite clearance time (by microscopy); PAHRP2: Plasmodium falciparum Histadine Rich Protein 2; PP: Per protocol; QN: Quinine; RBCs: Red blood cells; SD: Standard deviation; WBCs: White blood cells

\section{Acknowledgments}

We thank the people of Kingasani Community for participating in this trial, the staff of the KIMORU Research Centre for carrying out the project. We thank Mavuto Mukaka for his useful inputs during the statistical analysis and Naomi Waithira for her support with the data entry. We also thank Anna Jeffreys, Kate Rowlands and Christina Hubbart at the Wellcome Trust Centre for Human Genetics, University of Oxford, for genotyping the samples.

\section{Funding}

The study was supported by a grant from the Guilin Pharmaceutical company, which provided the artesunate but otherwise had no involvement in any part of the study. The genotyping undertaken at The Wellcome Trust Centre for Human Genetics was supported through a Wellcome Trust strategic award to the Resource Centre for Genomic Epidemiology of Malaria (090770/Z/09/Z) and core award (090532/Z/09/Z). This study was part of the Wellcome Trust Mahidol University Oxford Tropical Medicine Research Programme supported by the Wellcome Trust (106,698/Z/14/Z).

\section{Availability of data and materials}

The dataset and materials source of the current paper are not publicly available, but are available from the University of Oxford on reasonable request. 


\section{Authors' contributions}

Study design CF AD MO NW CW PB SJ ND; Data Collection CF MO; Data analysis SJL CF KS CW SS KC KR; Sample analysis SS, KC, CW, KR; Manuscript preparation CF AD CW SJL NW MO KR. All authors read and approved the final manuscript.

\section{Ethics approval and consent to participate}

The study was approved by the Oxford Tropical Medicine Ethics Committee and the University of Kinshasa, School of Public Health institutional review board. The study was explained to caregivers in French or Lingala and they were requested to sign a consent form to allow their child's participation in the study. The intervention was assigned by the study nurse, after the doctor confirmed eligibility and the caregivers had signed the informed consent.

\section{Consent for publication}

Not applicable.

\section{Competing interests}

The authors declare that they have no competing interests.

\section{Publisher's Note}

Springer Nature remains neutral with regard to jurisdictional claims in published maps and institutional affiliations.

\section{Author details}

Mahidol-Oxford Tropical Medicine Research Unit, Faculty of Tropical Medicine, Mahidol University, Bangkok, Thailand. ${ }^{2}$ Centre for Tropical Medicine and Global Health, Nuffield Department of Medicine, University of Oxford, Oxford, UK. ${ }^{3}$ Kinshasa School of Public Health, University of Kinshasa, Kinshasa, Democratic Republic of the Congo. ${ }^{4}$ Department of Clinical Tropical Medicine, Faculty of Tropical Medicine, Mahidol University, Bangkok, Thailand. ${ }^{5}$ Institut National de la Transfusion Sanguine, Université Paris Descartes/INSERM UMR_S 1134, Paris, France. 'Laboratoire d'Excellence GR-Ex, Paris, France. ${ }^{7}$ Assistance Publique-Hôpitaux de Paris, Centre National de Référence du Paludisme, Paris, France. ${ }^{8}$ Wellcome Trust Centre for Human Genetics, University of Oxford, Oxford, UK. ${ }^{9}$ WorldWide Antimalarial Resistance Network, Oxford, UK.

\section{Received: 3 March 2017 Accepted: 9 August 2017}

\section{Published online: 17 August 2017}

\section{References}

1. Dondorp AM, Fanello Cl, Hendriksen IC, Gomes E, Seni A, Chhaganlal KD Bojang K, Olaosebikan R, Anunobi N, Maitland K, et al. Artesunate versus quinine in the treatment of severe falciparum malaria in African children (AQUAMAT): an open-label, randomised trial. Lancet. 2010;376(9753):1647-57.

2. Dondorp A, Nosten F, Stepniewska K, Day N, White N, South East Asian Quinine Artesunate Malaria Trial g. Artesunate versus quinine for treatment of severe falciparum malaria: a randomised trial. Lancet. 2005;366(9487):717-25.

3. WHO. Management of Severe Malaria a Practical Handbook. 3rd ed. Geneva: WHO; 2013.

4. Mohapatra MK, Srinivas D, Kar AK, Murmu M. Anaphylactic reaction to intravenous artesunate. J Assoc Physicians India. 2009;57:183-4.

5. Leonardi E, Gilvary G, White NJ, Nosten F. Severe allergic reactions to oral artesunate: a report of two cases. Trans R Soc Trop Med Hyg. 2001;95(2):182-3.

6. Price $R$, van Vugt M, Phaipun L, Luxemburger C, Simpson J, McGready R, ter Kuile F, Kham A, Chongsuphajaisiddhi T, White NJ, et al. Adverse effects in patients with acute falciparum malaria treated with artemisinin derivatives. Am J Trop Med Hyg. 1999;60(4):547-55.

7. Rehman K, Lotsch F, Kremsner PG, Ramharter M. Haemolysis associated with the treatment of malaria with artemisinin derivatives: a systematic review of current evidence. Int J Infect Dis. 2014;29:268-73.

8. Rolling T, Agbenyega T, Krishna S, Kremsner PG, Cramer JP. Delayed haemolysis after artesunate treatment of severe malaria - review of the literature and perspective. Travel Med Infect Dis. 2015;13(2):143-9.

9. Phiri K, Esan M, van Hensbroek MB, Khairallah C, Faragher B, ter Kuile FO. Intermittent preventive therapy for malaria with monthly artemetherlumefantrine for the post-discharge management of severe anaemia in children aged 4-59 months in southern Malawi: a multicentre, randomised placebo-controlled trial. Lancet Infect Dis. 2012;12(3):191-200.
10. WHO. Guidelines for the treatment of malaria. 2nd ed. Geneva: WHO; 2010.

11. Angus BJ, Chotivanich K, Udomsangpetch R, White NJ. In vivo removal of malaria parasites from red blood cells without their destruction in acute falciparum malaria. Blood. 1997:90(5):2037-40.

12. Chong SS, Boehm CD, Higgs DR, Cutting GR. Single-tube multiplex-PCR screen for common deletional determinants of alpha-thalassemia. Blood. 2000;95(1):360-2.

13. MMV. Experts group meeting on delayed anaemia following treatment with injectable artesunate. Wien: MMV; 2013. https://www.mmv.org/sites/default/ files/uploads/docs/events/2013/InjectableArtesunateExpertGroupMeeting.pdf.

14. Flegg JA, Guerin PJ, White NJ, Stepniewska K. Standardizing the measurement of parasite clearance in falciparum malaria: the parasite clearance estimator. Malar J. 2011;10:339.

15. WHO: Haemoglobin concentrations for the diagnosis of anaemia and assessment of severity In., vol. WHO/NMH/NHD/MNM/11.12011.

16. NIAID/DAIDS: Table for grading the severity of adult and pediatric adverse events. 2014.

17. Jaureguiberry S, Ndour PA, Roussel C, Ader F, Safeukui I, Nguyen M, Biligui S, Ciceron L, Mouri O, Kendjo E, et al. Postartesunate delayed hemolysis is a predictable event related to the lifesaving effect of artemisinins. Blood. 2014;124(2):167-75.

18. Looareesuwan S, Davis TM, Pukrittayakamee S, Supanaranond W, Desakorn V, Silamut K, Krishna S, Boonamrung S, White NJ. Erythrocyte survival in severe falciparum malaria. Acta Trop. 1991:48(4):263-70.

19. Newton PN, Chotivanich K, Chierakul W, Ruangveerayuth R, Teerapong P, Silamut K, Looareesuwan S, White NJ. A comparison of the in vivo kinetics of plasmodium falciparum ring-infected erythrocyte surface antigen-positive and -negative erythrocytes. Blood. 2001;98(2):450-7.

20. Cao XT, Bethell DB, Pham TP, Ta TT, Tran TN, Nguyen TT, Pham TT, Nguyen TT, Day NP, White NJ. Comparison of artemisinin suppositories, intramuscular artesunate and intravenous quinine for the treatment of severe childhood malaria. Trans R Soc Trop Med Hyg. 1997;91(3):335-42.

21. Bunnag D, Viravan C, Looareesuwan S, Karbwang J, Harinasuta T. Clinical trial of artesunate and artemether on multidrug resistant falciparum malaria in Thailand. A preliminary report. Southeast Asian J Trop Med Public Health. 1991;22(3):380-5.

22. Rolling T, Agbenyega T, Issifou S, Adegnika AA, Sylverken J, Spahlinger D, Ansong D, Lohr SJ, Burchard GD, May J, et al. Delayed hemolysis after treatment with parenteral artesunate in African children with severe malaria-a double-center prospective study. J Infect Dis. 2014;209(12):1921-8.

23. Leowattana W, Krudsood S, Tangpukdee N, Brittenham G, Looareesuwan S. Defective erythropoietin production and reticulocyte response in acute plasmodium falciparum malaria-associated anemia. Southeast Asian J Trop Med Public Health. 2008:39(4):581-8.

24. Hendriksen IC, White LJ, Veenemans J, Mtove G, Woodrow C, Amos B, Saiwaew S, Gesase S, Nadjm B, Silamut K, et al. Defining falciparum-malariaattributable severe febrile illness in moderate-to-high transmission settings on the basis of plasma PfHRP2 concentration. J Infect Dis. 2013:207(2):351-61.

\section{Submit your next manuscript to BioMed Central and we will help you at every step:}

- We accept pre-submission inquiries

- Our selector tool helps you to find the most relevant journal

- We provide round the clock customer support

- Convenient online submission

- Thorough peer review

- Inclusion in PubMed and all major indexing services

- Maximum visibility for your research

Submit your manuscript at www.biomedcentral.com/submit
Biomed Central 